[108] Slutzker, B., H. C. Krolt, F. E. Ellis, and I. A. Silverstone: Pulmonary alveolar proteinosis: Case report and review of literature. Arch. intern. Med. 107, 264 (1961).

[109] -, and P. H. Perryman: Pulmonary alveolar poteinosis: Response to nebulized enzyme therapy. Arch. intern. Med. 109, 406 (1962).

[110] SNider, Th. H., Fr. M. Wilner, and B. M. Lewis: Cardiopulmonary physiology in a case of pulmonary alveolar proteinosis. Ann. intern. Med. 52, 1318 (1960).

[111] StansmFer, P. H. D., and C. Bodragors: Pulmonary alveolar proteinosis. Amer. J. clin. Path. 44, 539 (1965).

[112] Stern, H., W. F. Bond, and N. C. LaIos: Pulmonary alveolar proteinosis: A case report. Dis. Chest 39, 82 (1961).

[113] Taleghanr-Far, M., J. B. Barber, C. Sampson, and K. A. Harden: Cerebral nocardiosis and alveolar proteinosis. Amer. Rev, resp. Dis. 89, 561 (1964).

[114] Taxay, E. P., R. D. Montgomfry, and D. M. Wintsh: Studies of pulmonary alveolar microlithiasis and pulmonary alveolar proteinosis. Amer. J. clin. Path. 34, 532 (1960).

[115] Thsseradx, H.: Uber pulmonale alveolüre Proteinose (Lipoproteinose). Zbl. allg. Path. path. Anat. 106, 56 (1964).

[110] TruLING, W. und G. SEverIN: Zum Krankheitsbild der Mikrolithiasis alveolaris pulmonum. Med. Klin. 56, 51 (1961).

[117] UdanI, P. M., and S. MokgRJI: Pulmonary alveolar proteinosis. Indian J, Child, HIth 12, 256 (1963).

[118] Uenminger, E.: Mikrolithiasis, Amyloidose und Proteinose der Lungen. Beitr. Klin. Tuberk. 132, 130 (1965).

[119] Wegmans, T.: Blastomykose und andere Pilzerkrankungen der Lunge. Dtsch. Arch. klin. Med. 199, 192 (1952).

[120] Weylmawn, W.T.: Case report Nr. 27/1964 of the Massachusetts General Hospital. New. Engl. J. Med. 270, 1242 (1964).

[121] Winliams, G. E. Gr., D. R. K. Medley, and R. Brown: Pulmonary alveolar proteinosis. Lancet $\mathrm{I}, 1960,1385$.

[122] WITT, R., G. L. BAUM, and F. TARABE: Pulmonary alveolar proteinosis: A report of two cases with physiological data. Ohio St. med. J. 59, 1104 (1963).

[123] WoLmax, L.: The cerebral complications of pulmonary alveolar proteinosis. Lancet II, $1961,733$.

[124] Wukвтісн, S.: Pulmonale alveoläre Proteinose. Frankfurt. Z. Path. 72, 571 (1963).

Dr. med. H. vow EgIDY,

Priv. Doz. Dr. med. R. BässLer und

Prof. Dr. med. W. Turung

Universitätskliniken

65 Mainz, Langenbeckstraße 1

\title{
Berichtigung
}

\section{Spätergebnisse nach einer Lungenresektion wegen Tuberkulose}

\section{Mitteilung}

P. G. Schmidt und H. W. Ravch

Beitr. Klin. Tuberk. 183, 148-180 (1966)

Auf Seite 155 sind in Tabelle 1 in der 1. Spalte "Zahl" die beiden Zahlen falsch angegeben. Es muß richtig heißen: 